\title{
Joining end-to-end tubing of dissimilar materials by forming
}

\author{
Alves, L.M.; Nielsen, Chris Valentin; Silva, C.M.A.; Martins, P.A.F.
}

Published in:

International Journal of Pressure Vessels and Piping

Link to article, DOI:

10.1016/j.ijpvp.2016.11.006

Publication date:

2017

Document Version

Peer reviewed version

Link back to DTU Orbit

Citation (APA):

Alves, L. M., Nielsen, C. V., Silva, C. M. A., \& Martins, P. A. F. (2017). Joining end-to-end tubing of dissimilar materials by forming. International Journal of Pressure Vessels and Piping, 149, $24-32$.

https://doi.org/10.1016/j.jpvp.2016.11.006

\section{General rights}

Copyright and moral rights for the publications made accessible in the public portal are retained by the authors and/or other copyright owners and it is a condition of accessing publications that users recognise and abide by the legal requirements associated with these rights.

- Users may download and print one copy of any publication from the public portal for the purpose of private study or research.

- You may not further distribute the material or use it for any profit-making activity or commercial gain

- You may freely distribute the URL identifying the publication in the public portal

If you believe that this document breaches copyright please contact us providing details, and we will remove access to the work immediately and investigate your claim. 


\title{
JOINING END-TO-END TUBING OF DISSIMILAR MATERIALS BY FORMING
}

\author{
L.M. Alves ${ }^{a}$, C.V. Nielsen ${ }^{b}$, C.M.A. Silva ${ }^{a}$ and P.A.F. Martins ${ }^{a^{*}}$ \\ ${ }^{a}$ IDMEC, Instituto Superior Técnico, Universidade de Lisboa, Av. Rovisco Pais, 1049-001 Lisboa, \\ Portugal \\ ${ }^{b}$ Department of Mechanical Engineering, Technical University of Denmark, Produktionstorvet 425, 2800 \\ Kgs. Lyngby, Denmark
}

(*) Corresponding author. E-mail: pmartins@ist.utl.pt First author. E-mail: luisalves@ist.utl.pt Second author. E-mail: cvni@mek.dtu.dk Third author. E-mail: carlos.alves.silva@ist.utl.pt 


\section{ABSTRACT}

This paper presents a plastic deformation process for joining two tubes made from dissimilar materials by their ends. The process consists of two elementary tube forming operations that are carried out in a single stroke; expansion to produce two adjacent counterfacing surfaces and compression beading to lock the tubes together, and has potential to replace existing solutions based on the utilization of fastened, crimped, welded, brazed or adhesive bonded joints.

The investigation combines independent characterization of the materials, experimentation in a laboratory tool system and finite element modelling. Results give emphasis to the modes of deformation and failure that are used to setup the process window and demonstrate the simplicity and effectiveness of the proposed joining process for connecting carbon and stainless steel tubes by their ends. Additional results in aluminium and carbon steel tubes that were obtained by means of a two-stroke variant of the process confirm its potential for joining two tubes made from dissimilar materials with different strengths by their ends.

Keywords: Tubes, Joining by plastic deformation, Experimentation, Finite element method 


\section{INTRODUCTION}

In the state-of-the-art review on joining by forming, Mori et al. [1] concluded that application of plastic deformation is an increasingly useful solution to meet the growing demands of high productivity, low cost material versatility, high strength and environmental friendliness. Very recently, Groche et al. [2] performed a comprehensive analysis of the basic principles of joining by forming and highlighted future trends based upon ongoing research activities. They concluded that joining processes based on plastic deformation of at least one joining partner promise great potential regarding the production of multi-material joints and hybrid components consisting of dissimilar materials.

In the past months, Gonçalves et al. [3, 4] developed innovative plastic deformation solutions for joining tubes to sheets and for producing tube attachments of dissimilar materials at an angle to the axis of the main tube. The solutions are based on a combination of plastic instability and locking by means of compression beading, and a patent application by a manufacturer of automotive components protects the utilization of these solutions for connecting the lever and the fulcrum of a car hand-brake system [5]. However, the challenge of joining by plastic deformation two tubes made from dissimilar materials by their ends remained up to now unsolved, despite its great relevance in industrial applications comprising pipe lines, air-conditioning, refrigeration, heat exchangers and lightweight structures, among others.

Currently available technologies for joining two tubes by their ends (or near-ends) make use of welded, brazed, adhesive bonded, fastened or crimped joints (Fig. 1) but the number of realistic options is limited when the tubes to be joined are made from dissimilar materials. In fact, joining carbon and stainless steel tubes by welding, for example, may experience bimetallic corrosion at the welds when subjected to aggressive 
moist environments and often requires coating the weld seam by painting so that galvanic corrosion cells cannot be setup across the joint, where there is a composition gradient. Joining steel and aluminium tubes is even more complicated due to formation of very brittle intermetallic compounds and generally require the utilization of bimetallic transition inserts at the tube ends or the coating of the steel tube end with aluminium (by hot dip aluminizing or brazing), prior to welding [6].

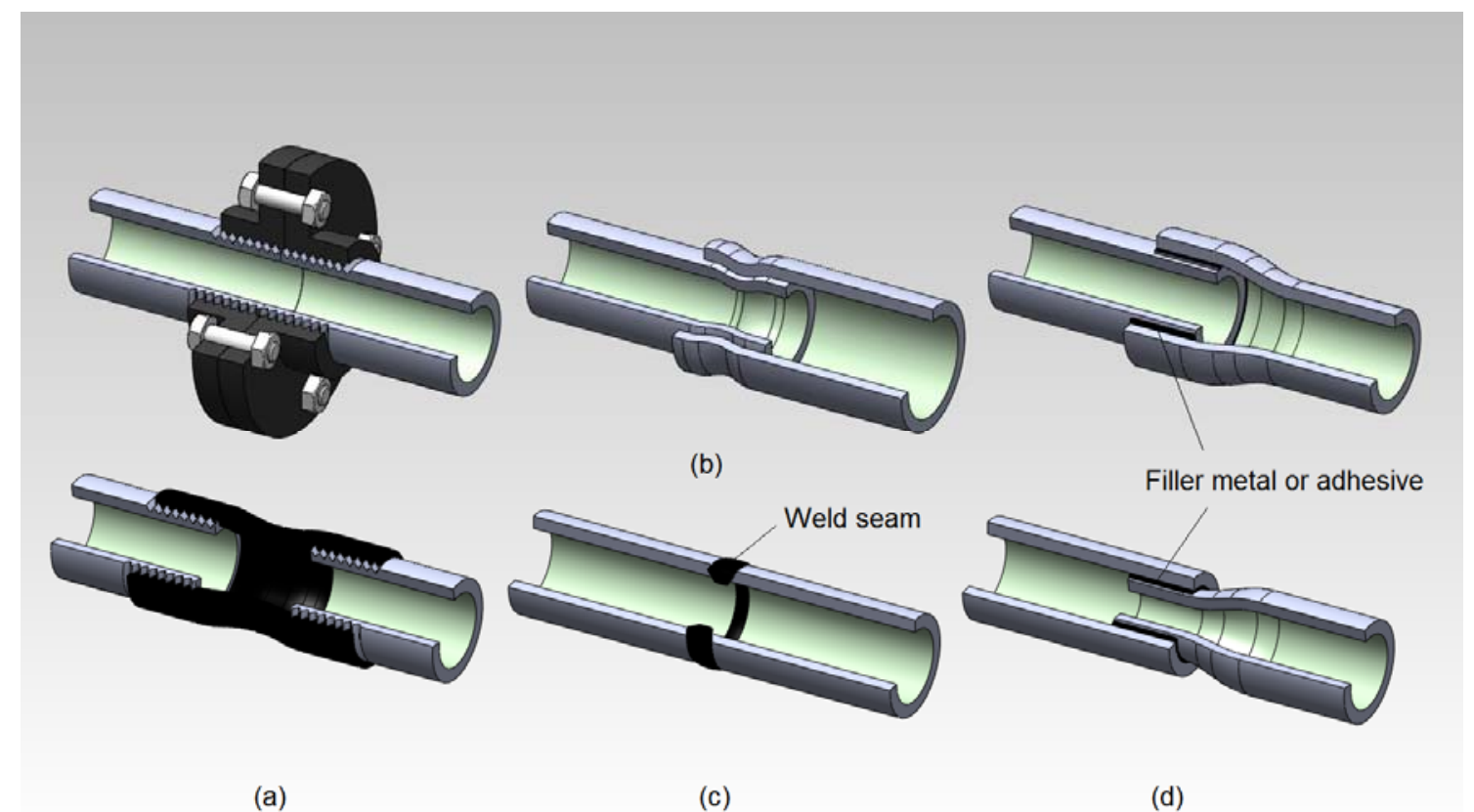

Fig. 1 - Currently available technologies for joining end-to-end tubing: (a) fastened joints with flanges and bulkhead unions, (b) crimped joints, (c) welded joints and (d) brazed or adhesive bonded joints.

The difficulties of welding two tubes by their ends become even more complicated in case of applications involving thin-walled tubes of dissimilar materials, like those shown in this paper. Brazing can be a valuable alternative to welding in case of thinwalled tubes made from dissimilar materials but it is not recommended for applications involving exposure to elevated service temperatures because the differences in thermal expansion rates of the tubes and fillers can give rise to thermal fatigue cracking. Brazing 
is also not recommended when full mechanical strength is required for the end-to-end tube joints [6].

The alternative to welding and brazing provided by adhesive bonding prevents the heating-cooling cycles but it is not a good option when high productivity is required. This is because adhesive bonding requires careful preparation with tight tolerances of the counterfacing tube surfaces and enough time for the adhesive to cure during which the joint must be kept immobilized until complete solidification. In addition, adhesive bonding may also experience decrease in performance over time under adverse environmental conditions [7].

From what was mentioned before, it may be concluded that currently available technologies for joining two tubes made from dissimilar materials by their ends are limited to mechanical fastening and crimping. Fastened joints (with flanges or bulkhead unions) make use of threads, screws and bolts, and are simple to design, easy to assemble and disassemble and available in standard sizes [8]. However, they suffer from aesthetic, geometric and dimensional limitations.

In contrast, crimped joints obtained by reduction, swaging [9] or electromagnetic forming [10] are not constrained by aesthetics or by the availability of flanges or bulkhead unions in standard sizes but are limited by the required mechanical strength and water or gas tightness. In case of crimped joints produced by electromagnetic forming there are additional demands for high electrical conductivity of the materials to be joined.

In a recent paper authors proposed a new plastic deformation process for joining two tubes by their ends and discussed its applicability to commercial carbon steel tubes [11]. The process is schematically shown in Fig. 2 and consists of two forming stages carried out in a single stroke. The first stage (hereafter referred to as 'the expansion stage') 
produces the adjacent counterfacing surfaces of the tubes to be joint by forcing the upper tube against the chamfered end of the lower tube in order to radially expand the initial unsupported height $l_{o}$ of the upper tube. During this stage the chamfered end of the lower tube acts like a tapered punch. The second stage starts when the upper tube gets into contact with the lower die (or, alternatively, when the lower tube gets into contact with the inner surface of the upper tube) and creates the lock between the adjacent counterfacing surfaces of the two tubes by compression beading.

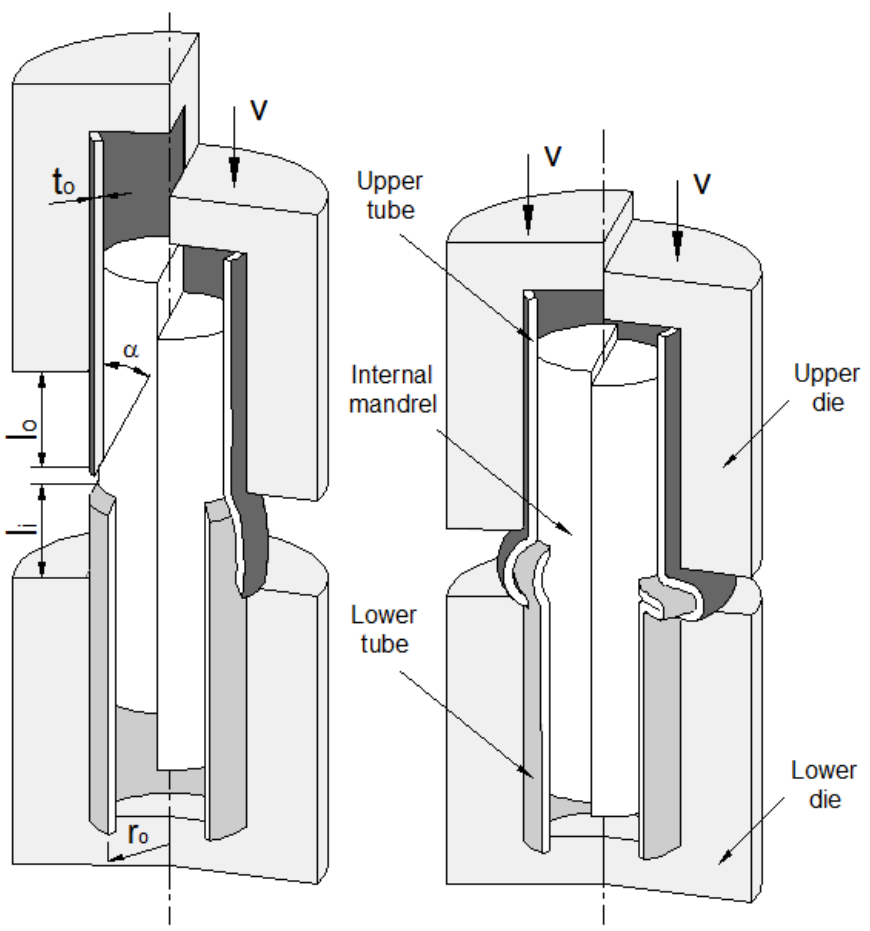

(a)

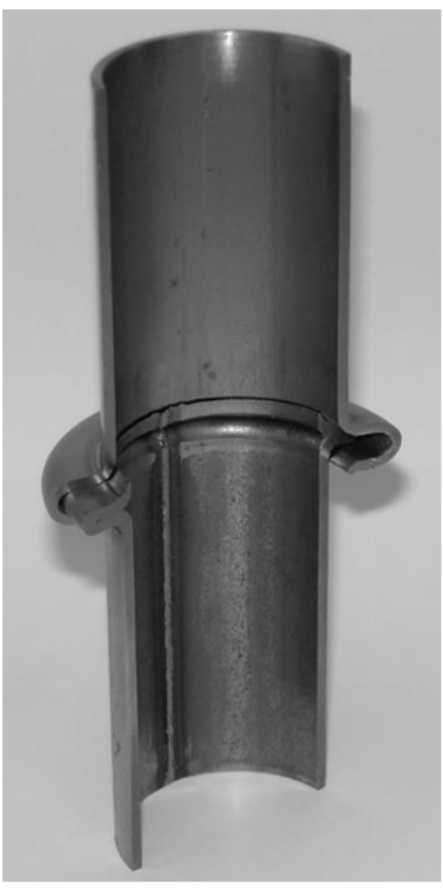

(b)

Fig. 2 - Joining end-to-end tubing by forming.

(a) Schematic representation of the process and notation;

(b) Photograph showing a detail of the cross section of a stainless steel tube (top) joined by its end to a carbon steel tube (bottom).

This paper explores the possibility of connecting two tubes of dissimilar materials by their ends using the abovementioned plastic deformation process. The motivation to take a step forward derives from the fact that previous investigation on deformation modes and process window was not influenced by differences in the plastic behaviour 
of the mating tubes to be joined because it was limited to commercial carbon steel tubes.

Under these circumstances, the aim and objectives of this paper are the following: (i) to revisit the previously observed modes of deformation in the light of the plastic behaviour of stainless steel tubes, (ii) to investigate the influence of the relative position between the counterfacing surfaces of carbon and stainless steel tubes on the resulting end-to-end joints, (iii) to set-up a process window for joining carbon and stainless steel tubes by their ends and (iv) to present a two stroke variant of the process for joining tubes of dissimilar materials with very different strengths (e.g. aluminium and carbon steel tubes).

The organization of the paper is the following. Section 2 summarizes the mechanical characterization of the carbon steel and stainless steel tubes, presents the fundamentals of the plastic deformation process for joining two tubes of dissimilar materials by their ends and provides information on the experimental work plan. Section 3 provides insight on the finite element simulation conditions with special emphasis on contact modelling along the counterfacing surfaces of the two tubes to be joined. Section 4 presents and discusses the results obtained, namely the modes of deformation and the influence of the major operating parameters on the process window and on the overall force requirements. Section 5 concludes. 


\section{EXPERIMENTATION}

\subsection{Mechanical characterization of the material}

The investigation was performed on commercial S460MC (carbon steel) and AISI304 (stainless steel) welded tubes in the 'as-received' condition.

The stress-strain behaviour of the tubes was determined by means of tensile and stack compression tests carried out at room temperature. The tensile test specimens were machined from the supplied tube stock and the stack compression test specimens were assembled by pilling up circular discs cut from the tube stock by a hole-saw.

The tests were performed at room temperature on a universal testing machine with a cross-head speed equal to $100 \mathrm{~mm} / \mathrm{min}(1.7 \mathrm{~mm} / \mathrm{s})$ and the resulting stress-strain curves were approximated by the following Ludwik-Hollomon’s equations,

$$
\begin{gathered}
\sigma=616 \varepsilon^{0.06}(\mathrm{MPa})-\mathrm{S} 460 \mathrm{MC} \\
\sigma=1266 \varepsilon^{0.34}(\mathrm{MPa})-\mathrm{AISI} 304
\end{gathered}
$$

Plastic instability of S460MC and AISI304 tubes was characterized by compressing S460MC and AISI304 tubular specimens with a reference radius $r_{0}=16 \mathrm{~mm}$ and a wall thickness $t_{0}=1.5 \mathrm{~mm}$ between flat parallel compression platens. The experimental value of the critical load $P_{c r}$ that was needed for triggering plastic instability waves (or wrinkles) along the tubes was $93.5 \mathrm{kN}$ and $79 \mathrm{kN}$ in case of S460MC and AISI304 tubes, respectively.

Further information on the methods and procedures that are utilized to obtain the stressstrain curves and the critical instability loads of tubes is available elsewhere [12]. 


\subsection{Working methods and procedures}

The laboratory tool system for joining tubes of dissimilar materials by their ends was installed in the hydraulic testing machine (Instron SATEC $1200 \mathrm{kN}$ ) where the mechanical characterization of the tubes had previously been performed. Five major operating parameters are identified in Fig. 2a; (i) the reference radius $r_{0}$ of the tubes, (ii) the thickness $t_{o}$ of the tube wall, (iii) the angle $\alpha$ of the chamfered tube ends, (iv) the initial unsupported height $l_{o}$ of the upper tube and (v) the initial unsupported height $l_{i}$ of the lower tube.

The experiments were carried out at room temperature on S460MC and AISI304 tubes with a reference radius $r_{0}=16 \mathrm{~mm}$, a wall thickness $t_{0}=1.5 \mathrm{~mm}$, a tube end chamfered angle $\alpha=25^{\circ}$ and a velocity equal to $100 \mathrm{~mm} / \mathrm{min}(1.7 \mathrm{~mm} / \mathrm{s})$. The initial unsupported heights $\left(l_{o}\right.$ and $\left.l_{i}\right)$ of the upper and lower tubes were allowed to vary between $10 \mathrm{~mm}$ and $25 \mathrm{~mm}$ and the aspect ratio $l_{o} / l_{i}=1$ due to accumulated knowledge from previous experiments performed in S460MC tubes [11].

The work plan was structured in two complementary parts. Firstly, the end-to-end joining of tubes of the same material by retrieving data from previous work on S460MC tubes [11] and by performing new experiments in AISI304 tubes. Secondly, the end-toend joining of tubes of dissimilar materials by performing new experiments with S460MC and AISI304 tubes. The experiments in tubes of dissimilar materials were planned upon intersection of the individual process windows of the end-to-end joining of S460MC and AISI304 tubes. The process windows are characterized by the slenderness ratio $l_{\text {gap }} / r_{0}$ between the initial unsupported gap height $\left(l_{\text {gap }}=l_{o}+l_{i}\right)$ and the reference radius $r_{0}$ of the tubes. 
Table 1 summarizes the work plan. The experiments were done in a random order and at least two repetitions were made for each test case.

In addition to this, authors performed experiments on a variant of the proposed joining process that was specially developed to connect tubes of dissimilar materials (carbon steel S460MC and aluminium AA6060-T6) with very different strengths. The results of these experiments are addressed at the end of the presentation.

\begin{tabular}{|c|c|c|c|c|c|}
\hline Test case & Material & $l_{o} \mathbf{( m m )}$ & $l_{i} \mathbf{( m m )}$ & $l_{\text {gap }}(\mathbf{m m})$ & $l_{\text {gap }} / r_{0}$ \\
\hline 1 & AISI304 & 10 & 10 & 20 & 1.25 \\
\hline 2 & AISI304 & 15 & 15 & 30 & 1.875 \\
\hline 3 & AISI304 & 20 & 20 & 40 & 2.5 \\
\hline 4 & AISI304 & 25 & 25 & 50 & 3.125 \\
\hline 5 & S460MC - AISI304 & 15 & 15 & 30 & 1.875 \\
\hline 6 & AISI304 - S460MC & 15 & 15 & 30 & 1.875 \\
\hline
\end{tabular}

Table 1 - The experimental work plan (nomenclature according to Fig. 2).

\section{FINITE ELEMENT MODELLING}

The numerical simulation of the joining process was performed with the finite element computer program I-form. The program was developed by the authors and is built upon the irreducible finite element flow formulation, which is based on the following variational principle (extended to account for contact and friction),

$$
\Pi=\int_{V} \bar{\sigma} \dot{\bar{\varepsilon}} d V+\frac{1}{2} K \int_{V} \dot{\varepsilon}_{v}^{2} d V-\int_{S_{T}} T_{i} u_{i} d S+\int_{S_{f}}\left(\int_{0}^{\left|u_{r}\right|} \tau_{f} d u_{r}\right) d S+\frac{1}{2} P \sum_{c=1}^{N_{c}}\left(g_{n}^{c}\right)^{2}+\frac{1}{2} \mu P \sum_{c=1}^{N_{c}}\left(g_{t}^{c}\right)^{2}
$$

where $\bar{\sigma}$ denotes the effective stress, $\dot{\bar{\varepsilon}}$ is the effective strain rate, $\dot{\varepsilon}_{V}$ is the volumetric strain rate, $K$ is a large positive constant imposing the incompressibility of volume $V$, $T_{i}$ and $u_{i}$ are the surface tractions and velocities on surface $S_{T}, \tau_{f}$ and $u_{r}$ are the 
friction shear stress and the relative velocity on the contact interface $S_{f}$ between tubes and tooling (dies and mandrel).

The last two terms in (2) account for contact between the counterfacing surfaces of the two tubes by penalizing normal gap velocity $g_{n}^{c}$, when otherwise leading to penetration, and by constraining tangential gap velocity $g_{t}^{c}$ to account for frictional effects. Gap velocities in each of the $N_{c}$ contact pairs are defined as,

$$
\begin{gathered}
g_{n}^{c}=\mathbf{v}_{p} \cdot \mathbf{n}-\beta \mathbf{v}_{1} \cdot \mathbf{n}-(1-\beta) \mathbf{v}_{2} \cdot \mathbf{n} \\
g_{t}^{c}=\mathbf{v}_{p} \cdot \mathbf{t}-\beta \mathbf{v}_{1} \cdot \mathbf{t}-(1-\beta) \mathbf{v}_{2} \cdot \mathbf{t}
\end{gathered}
$$

where nodal velocities $\mathbf{v}_{P}, \mathbf{v}_{1}$ and $\mathbf{v}_{2}$ are illustrated in Fig. 3, which schematically shows a contact pair consisting of a contacting nodal point $N_{P}$ and the nodal points of the opposing line segment $N_{1}$ and $N_{2}$ of an element of the counterfacing tube surface (or of the contacting tube surface itself due to self-contact in the later stages of deformation).

Fig. 3 also identifies the normal and tangential versors $\mathbf{n}$ and $\mathbf{t}$. The projection point of the contacting node on the opposing line segment, which is calculated based on the relative nodal velocities, is identified by the dimensionless parameter $\beta$ (Fig. 3a). Natural symmetry is obtained because nodes of the counterfacing element face can be contacting nodes in other contact pairs. The penalization of the normal gap velocity is ensured through a large positive number $P$ while the constraining of the tangential gap velocity takes place through penalization by $\mu P$. By doing this, the ratio of the tangential and the normal penalties is equal to $\mu$, which is used for calibration of the frictional effects between the tubes in analogy to Amonton-Coulomb friction. 


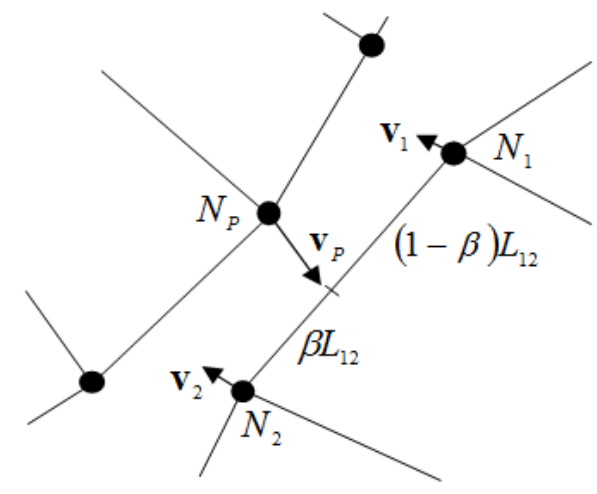

(a)

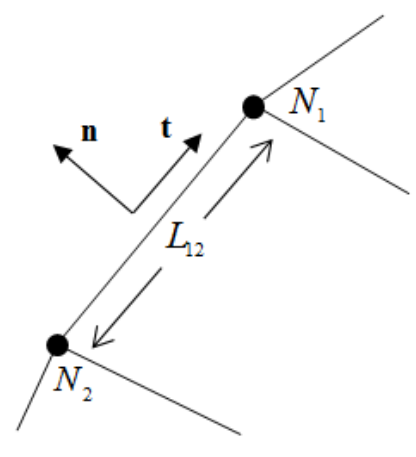

(b)

Fig. 3 - Schematic representation of the contact between the two tubes and associated notation.

(a) Boundary node $N_{P}$ contacting line segment $N_{1}-N_{2}$ due to nodal velocities $\mathbf{v}_{P}$, $\mathbf{v}_{1}$ and $\mathbf{v}_{2}$. The dimensionless parameter $\beta$ defines the projection point of the contacting node on the line segment;

(b) Identification of line segment length $L_{12}$ and versors $\mathbf{t}$ and $\mathbf{n}$ defining tangential and normal directions in the contact pair.

Further information on the finite element flow formulation and the computer program Iform can be found elsewhere [13].

The numerical simulation of the joining process was performed in two different stages corresponding to the previously mentioned elementary tube forming operations by expansion and compression beading. The tubes were discretized by means of axisymmetric linear quadrilateral elements (Fig. 4) on account of rotational symmetry and because no anisotropy effects due to material or welding seam were taken into consideration.

In contrast to what was performed in the aforementioned previous work on joining endto-end tubing of S460MC carbon steel tubes [11], the thickness direction was discretized by means of 10 axisymmetric linear quadrilateral elements in order to avoid intermediate remeshing operations and to successfully model the progressive distortion of the mesh from the expansion stage to subsequent plastic instability and locking by 
compression beading. The dies and mandrel are schematically shown in Fig. 4a and were discretized by means of contact-friction linear elements, again taking advantage of the rotational symmetry.

The overall CPU time for a typical analysis consisting of a mesh with approximately 2500 nodal points and 2200 linear quadrilateral elements was approximately equal to 5 min. on a standard laptop computer equipped with an Intel i7 CPU (2.7 GHz) processor.

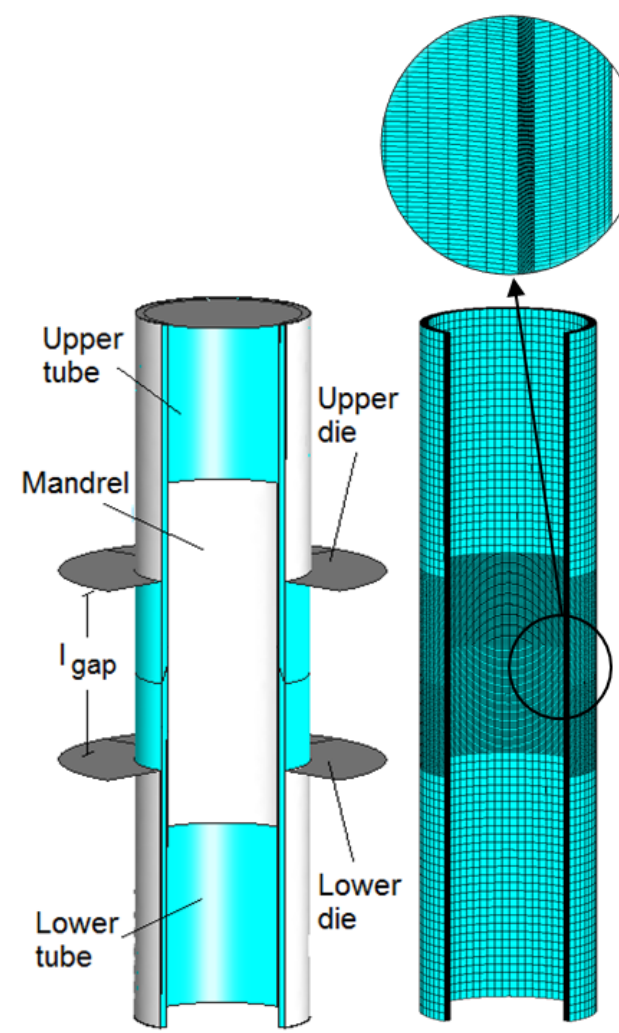

(a)

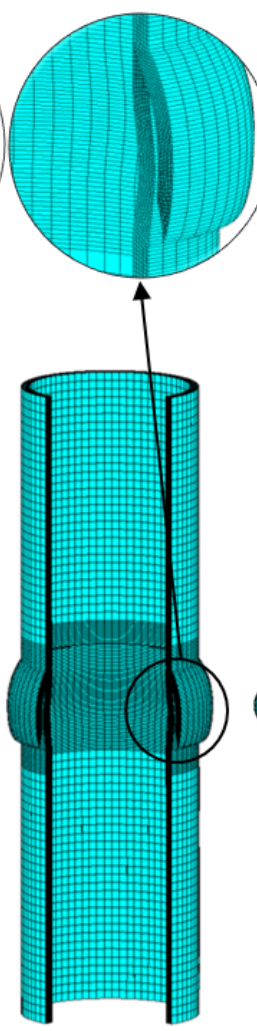

(b)
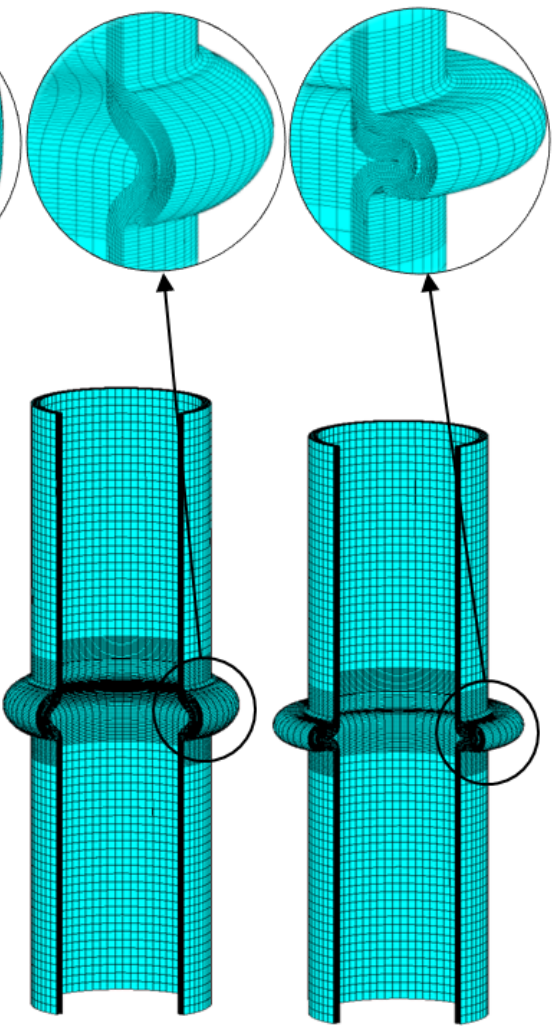

Fig. 4 - Numerical modeling of the joining end-to-end tubing by forming (case 2 of Table 1)

(a) Schematic representation showing the different entities that were utilized to model the tubes and the active tool parts;

(b) Finite element predicted geometry showing the expansion of the upper tube and the development of plastic instability and locking by compression beading between the upper and lower tubes. 


\section{RESULTS AND DISCUSSION}

\subsection{Modes of deformation}

Previous work on joining S460MC carbon steel tubes by their ends revealed the existence of three modes of deformation that are dependent on the slenderness ratio $l_{\text {gap }} / r_{0}$ (Fig. 5).

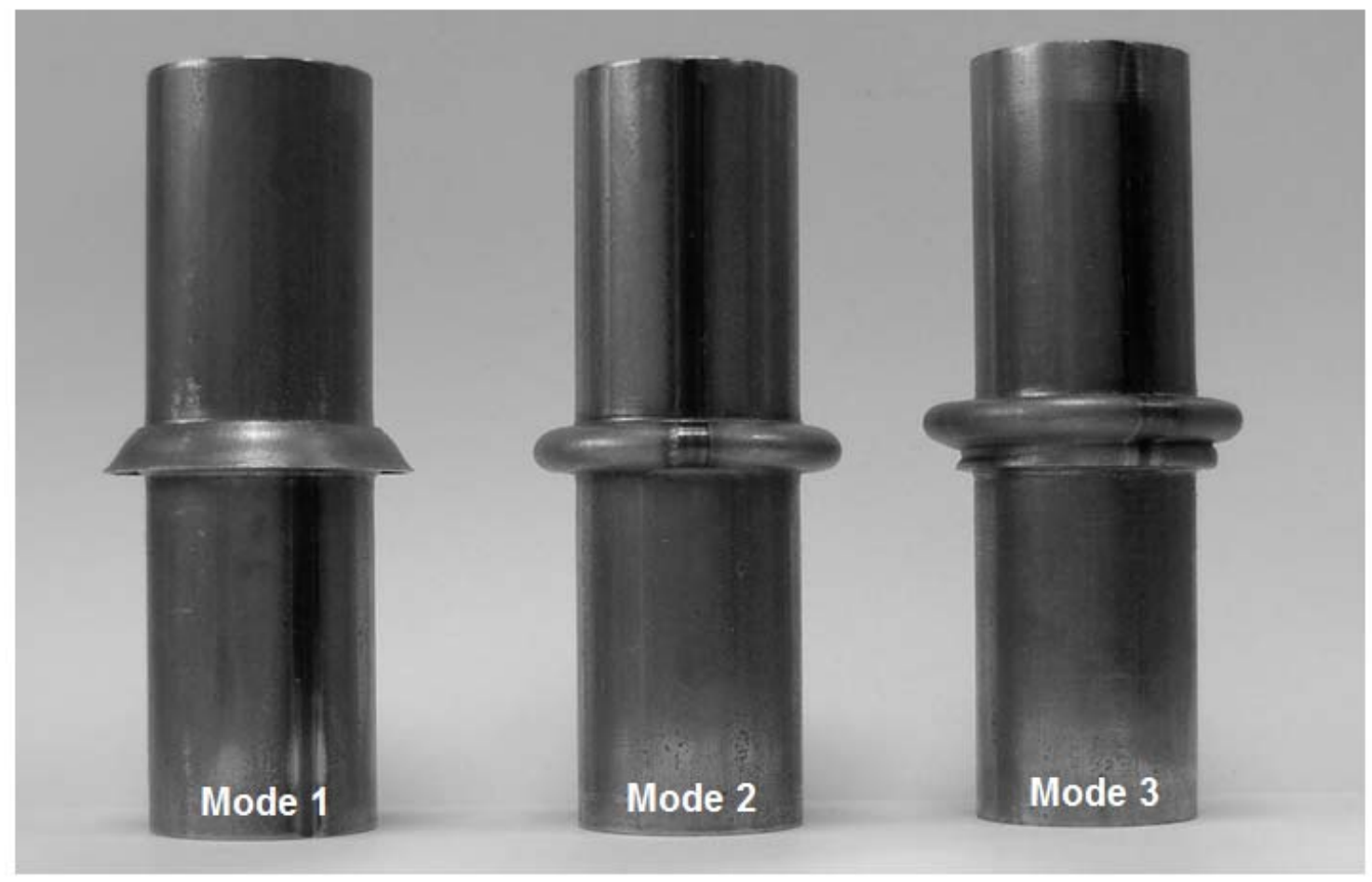

Fig. 5 - Typical modes of deformation (1, 2 and 3) that may develop during joining end-to-end tubing of S460MC by forming.

As seen in the leftmost test sample, below a threshold value $l_{\text {gap }} / r_{0}=1.25$ it is not possible to join the tubes by their ends because the gap opening $l_{\text {gap }}$ is not big enough for the counterfacing surfaces of the two tubes to be placed in the correct position for subsequent locking by compression beading. This mode of deformation will be hereafter referred to as 'mode 1 '.

Above a threshold value $l_{\text {gap }} / r_{0}=3.125$ there is a tendency to develop multiple plastic instability waves due to the outsized values of the unsupported free lengths of the upper 
and lower tubes. The second plastic instability wave may result either incomplete (as shown in the rightmost test sample of Fig. 5) or collide with the previously formed compression bead in order to form a poor quality, unacceptable, double joint, when the gap opening $l_{\text {gap }}$ becomes extremely large. This mode of deformation will be hereafter referred to as 'mode 3'.

For values of the slenderness ratio $1.25<l_{\text {gap }} / r_{0}<3.125$, compression beads are soundly triggered and good joints are formed between the ends of the S460MC tubes. This mode of deformation will be hereafter referred to as 'mode 2' and corresponds to the test sample placed in the middle of Fig. 5.

The quality and performance of the joints produced by deformation mode 2 was further evaluated by means of destructive tensile and water tightness tests. In case of the destructive tensile test, results show that the joint is capable of withstanding approximately $40 \mathrm{kN}$ of tensile load before failing by cracking (Fig. 6a). In case of the water tightness test, the build-up of pressure was accomplished through the displacement of two pistons inside the connected tubes up to a level of pressure of approximately $24 \mathrm{MPa}$ at which leakage started (Fig. 6b).

The experimental work performed with AISI304 stainless steel tubes revealed the aforementioned three modes of deformation plus one mode of failure that had not been previously seen in S460MC tubes. The mode of failure is due to meridional cracking along the plastically deformed weld seam and develops for values of the slenderness ratio $l_{\text {gap }} / r_{0} \geq 2.5$ (Fig. $7 \mathrm{a}$ ). The location of cracking corroborates with the finite element predictions of accumulated damage $D$ associated with the normalized Cockcroft-Latham ductile damage criterion [14], 


$$
D=\int_{0}^{\bar{\varepsilon}} \frac{\sigma_{1}}{\bar{\sigma}} d \bar{\varepsilon}
$$

that are included in Fig. 7b and allows determining the critical values of ductile damage $D_{\text {crit }}$ at the onset of cracking. As shown, accumulated values of ductile damage above $D_{\text {crit }}=0.58$ will trigger the opening of cracks by circumferential tensile stresses due to the poor ductility of the weld seam of the AISI304 tubes compared to that of the S460MC tubes.

The operative conditions for joining end-to-end tubing of dissimilar materials (S460MC and AISI304 tubes) were built upon the intersection of the individual process windows that were determined for each of the materials.

$$
\begin{gathered}
1.25<l_{\text {gap }} / r_{0}<3.125-\mathrm{S} 460 \mathrm{MC} \\
1.25<l_{\text {gap }} / r_{0}<2.5-\mathrm{AISI} 304
\end{gathered}
$$

Although the process window for successfully joining S460MC and AISI304 tubes by their ends is small, it does not mean that the process is limited to a reduced number of applications because a small window simply means that the unsupported heights of the upper and lower tubes must be controlled within a compact range of values in order to connect any two tubes by their ends.

Fig. 8 shows the experimental and finite element predicted modes of deformation that were obtained for a slenderness ratio $l_{\text {gap }} / r_{0}=1.875$. As seen in the figure, there is no influence of the relative position between the counterfacing surfaces of S460MC and AISI304 tubes. This is because the strengths of the carbon and stainless steel tubes are similar. As will be seen later in the presentation, tubes made from dissimilar materials with very different strength are not only sensitive to the relative position as they require alternative joining strategies based on a variant of the proposed process. 


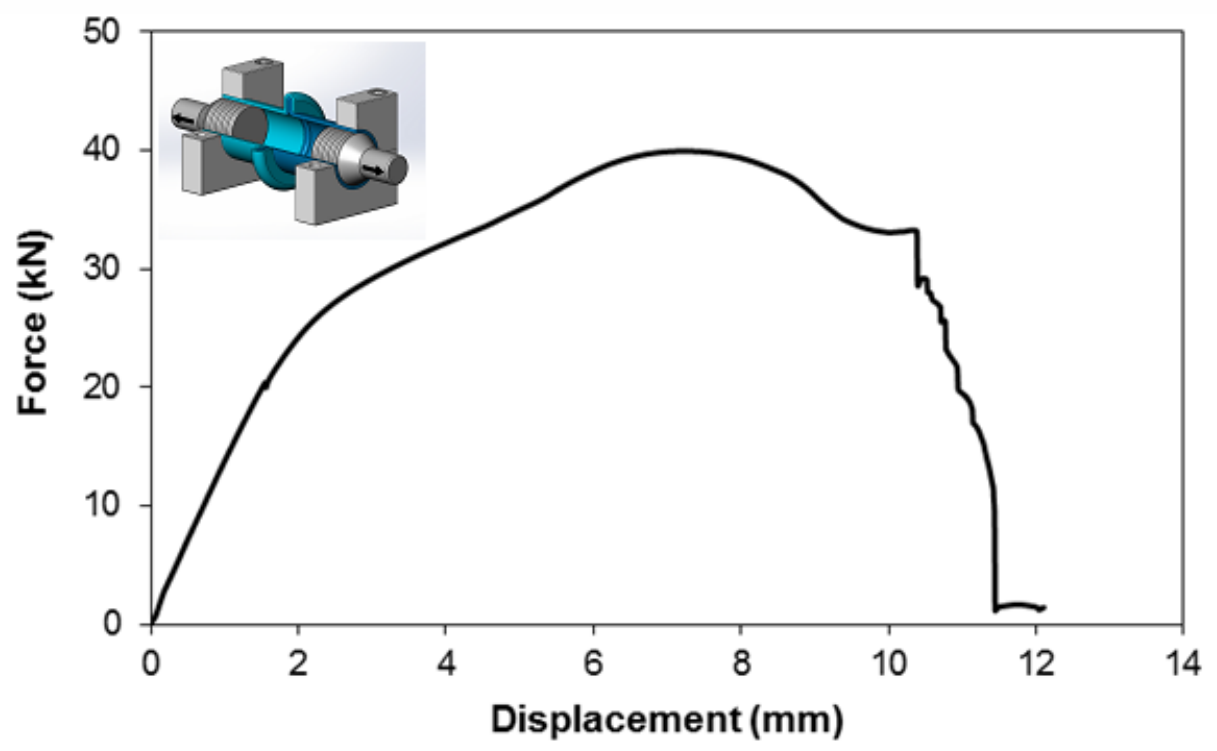

(a)

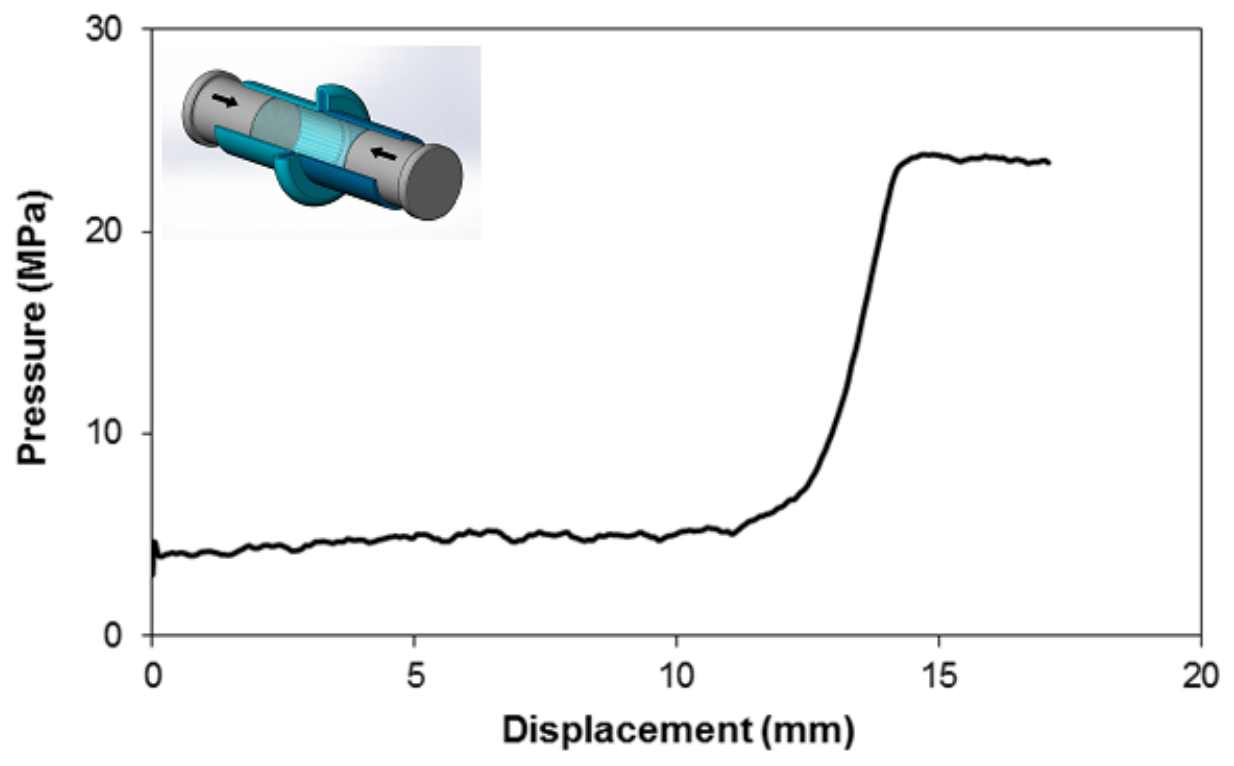

(b)

Fig. 6 - Destructive tests on end-to-end joints of S460MC tubes produced by deformation mode 2.

(a) Experimental evolution of the load-displacement curve during destructive tensile tests;

(b) Experimental evolution of pressure with displacement of the piston during water tightness tests. 


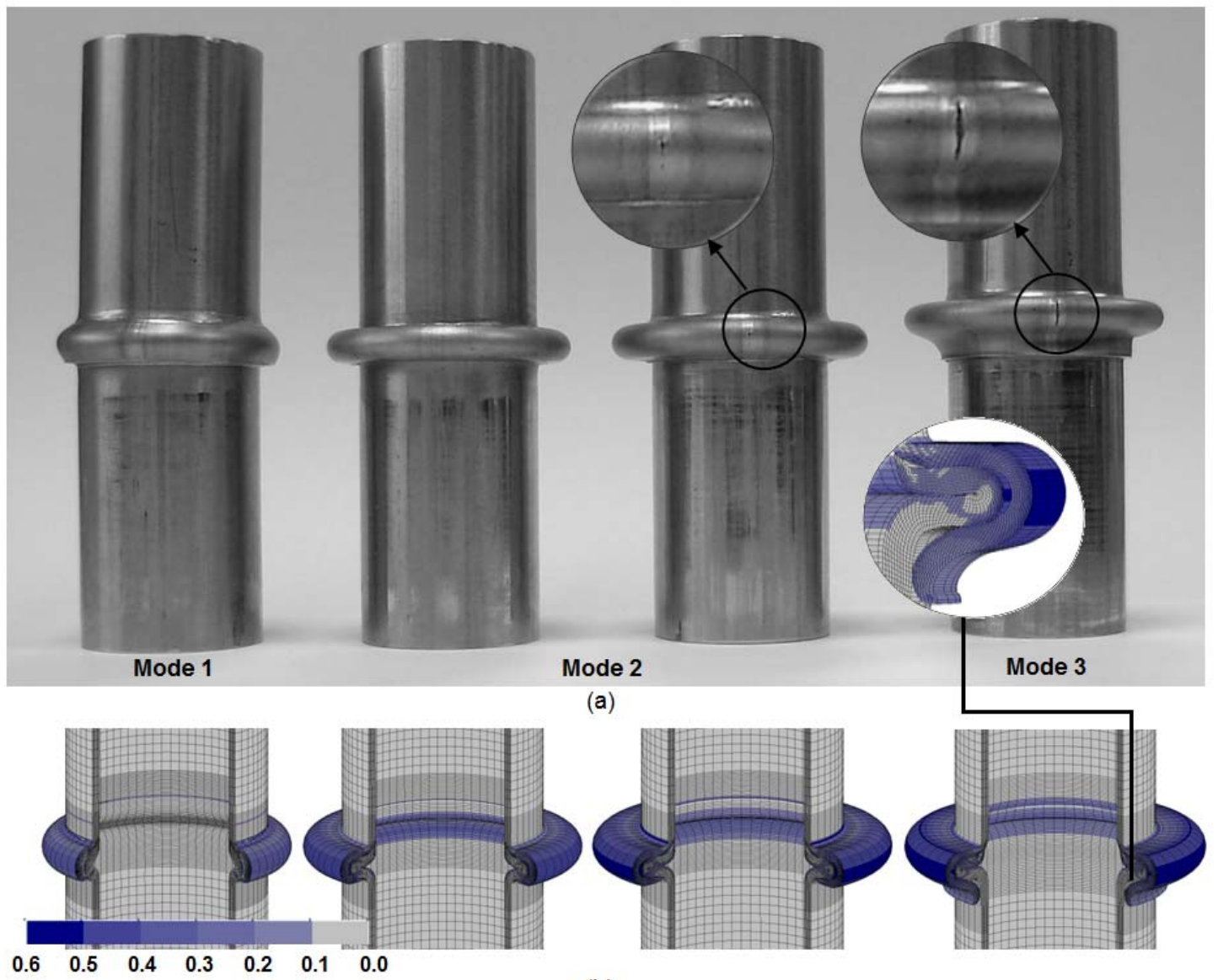

(b)

Fig. 7 - Joining end-to-end tubing of AISI304 stainless steel by forming.

(a) Typical modes of deformation (1, 2 and 3) and failure by cracking;

(b) Finite element predicted distribution of the accumulated damage according to the normalized Cockcroft-Latham ductile damage criterion.

\subsection{Load requirements}

Fig. 9 presents the experimental and finite element predicted evolution of the load with displacement for the testing conditions corresponding to joining end-to-end tubing of similar (AISI304 tubes, shown in Fig. 7) and dissimilar materials (AISI304 and S460MC tubes, shown in Fig. 8) by forming.

In case of joining AISI304 tubes (Fig. 9a), the overall trend of the load-displacement curve is identical to that previously observed with S460MC tubes [11]. The test cases giving rise to deformation mode 1 (e.g. case 1 in Fig. 9a) experience non-steady tube expansion in which, after an initial stage (labelled as ' $A$ '), there is a steep increase of 
the load (labelled as 'B') resulting from the unsupported region of the upper tube being forced into the chamfered edge of the lower tube in order to obtain circumferential stretching. The reason why the load grows above the critical value of load corresponding to simultaneous plastic instability of the upper and lower tubes (refer to the dashed horizontal line labelled as 'Total plastic instability' in Fig. 9a) is due to fact that the gap opening $l_{\text {gap }}$ is not big enough for triggering plastic instability and subsequent locking by compression beading.

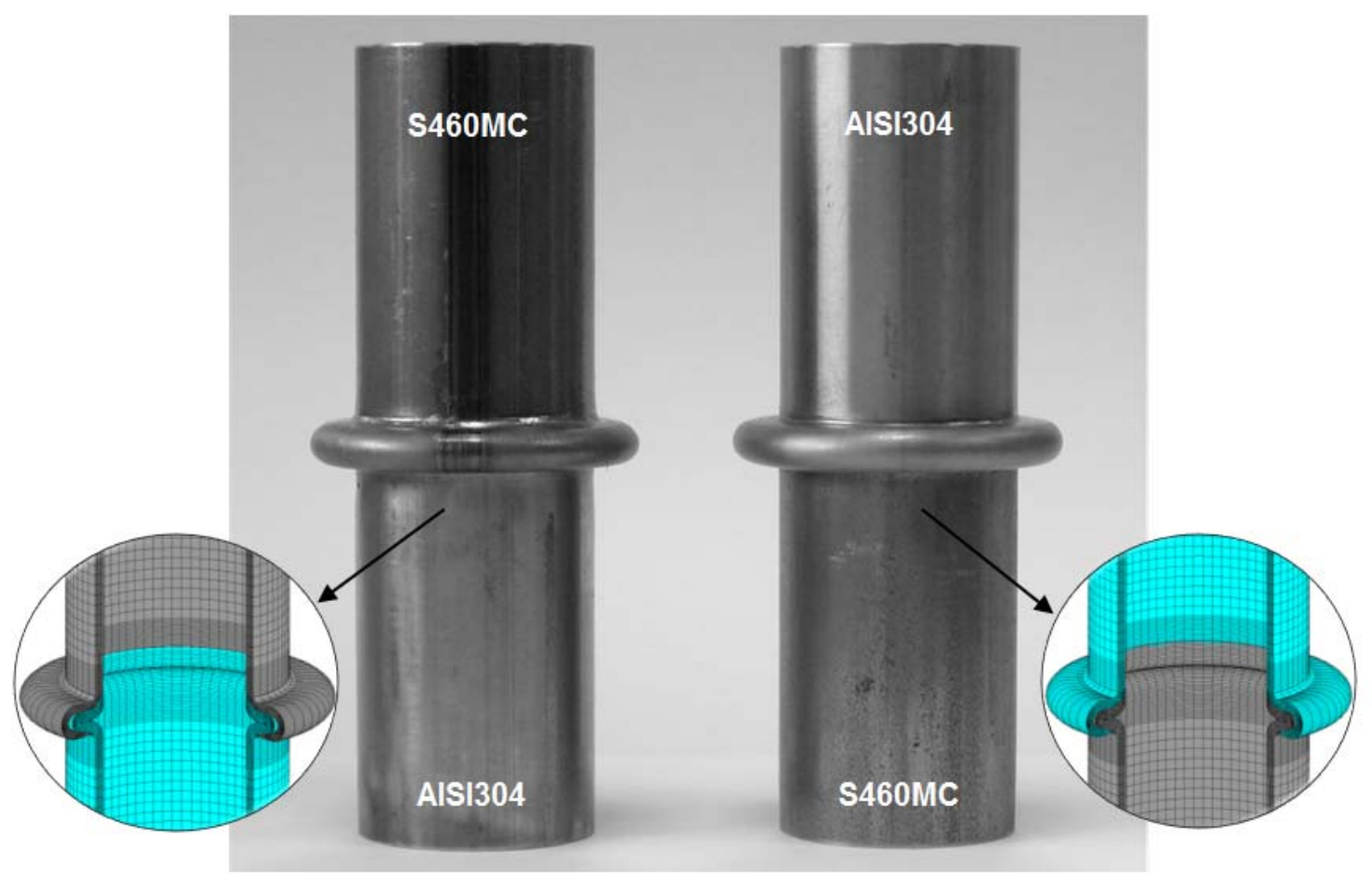

Fig. 8- Experimental and finite element computed geometries in joining end-to-end tubing of S460MC and AISI304 by forming (cases 5 and 6 of Table 1). 


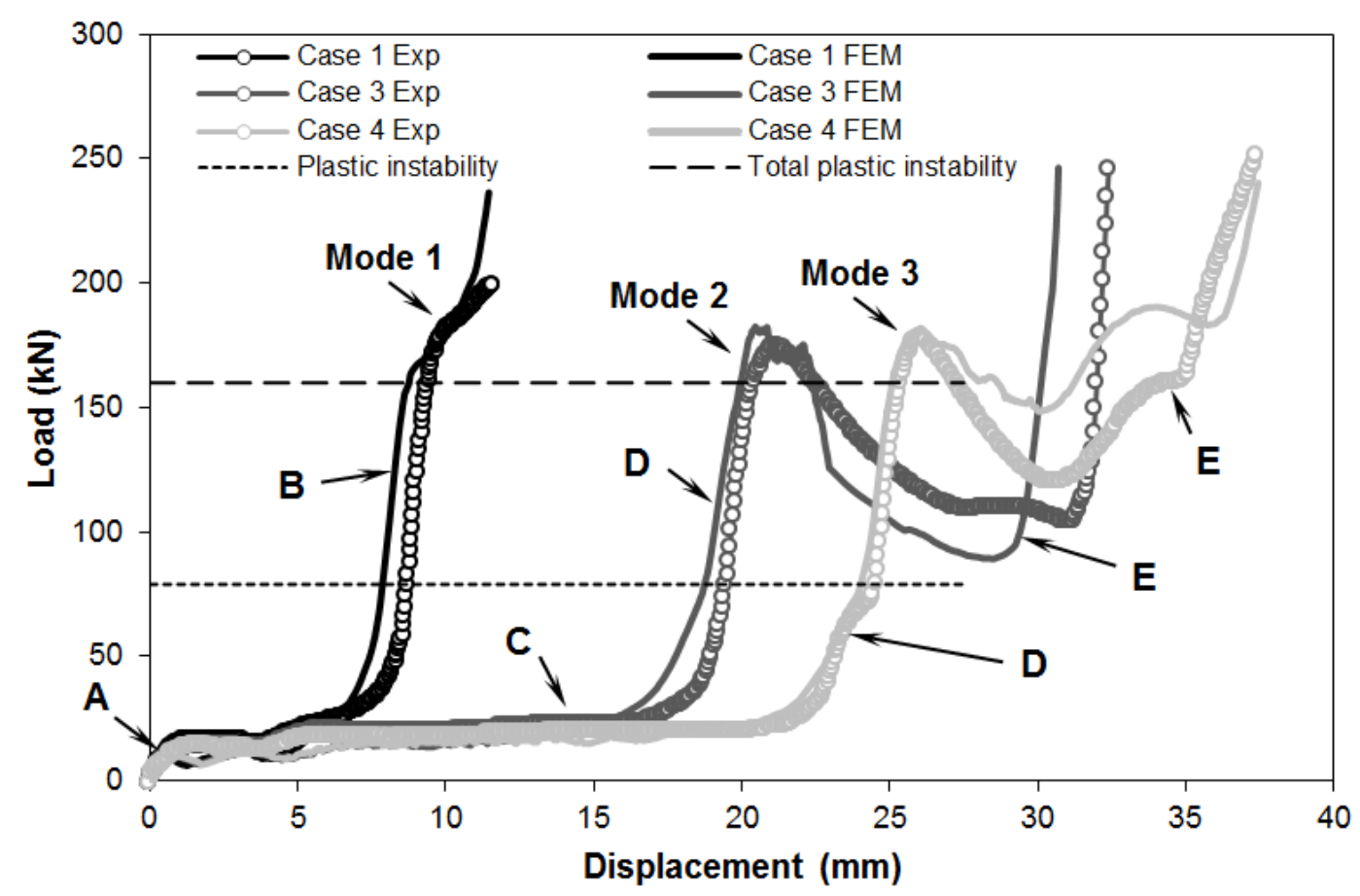

(a)

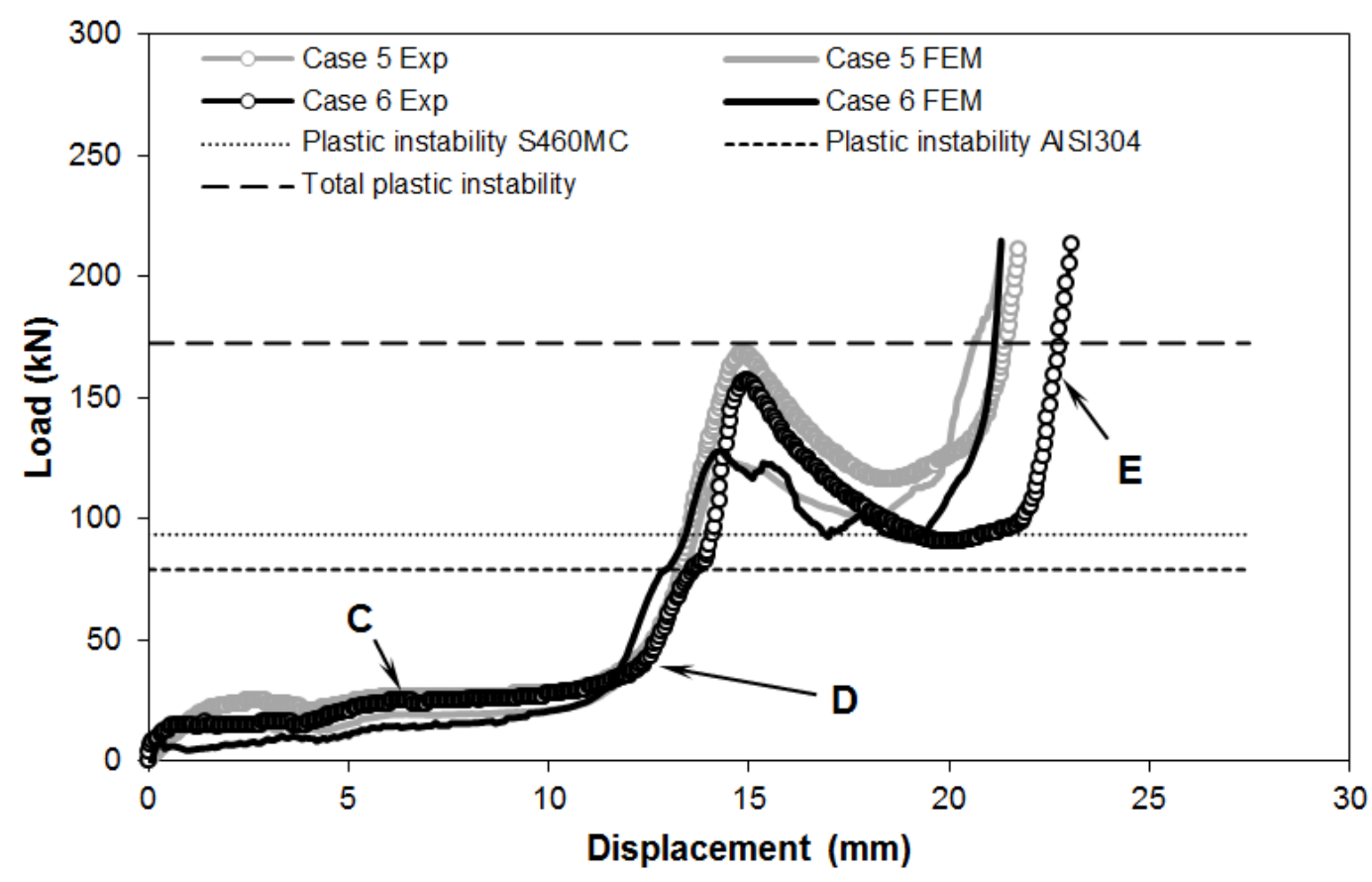

(b)

Fig. 9 - Experimental and finite element predicted evolution of the load-displacement curves for joining end-to-end tubing by forming.

(a) Cases 1, 3 and 4 of Table 1 (AISI304 tubes);

(b) Cases 5 and 6 of Table 1 (AISI304 and S460MC tubes). 
In contrast, the test cases giving rise to deformation modes 2 and 3 present an initial steady-state expansion stage (labelled as ' $\mathrm{C}$ ') during which the forming load is approximately constant (20 kN) and a second forming stage (labelled as 'D' and ' $\mathrm{E}$ ') characterized by plastic instability and locking by compression beading. Region ' $\mathrm{D}$ ' corresponds to a steep increase of the forming load up to a peek value of approximately $175 \mathrm{kN}$ whereas region ' $\mathrm{E}$ ' is linked to the subsequent decrease of the forming load due to the development of plastic instability and to the final upward tail of the loaddisplacement curve due to locking of the upper and lower tubes by compression beading.

In case of joining AISI304 and S460MC tubes (Fig. 9b) under a slenderness ratio $l_{\text {gap }} / r_{0}=1.875$ the general trend of the load-displacement curve is identical to that of mode 2. In fact, after the initial steady-state expansion stage (labelled as ' $C$ ') during which the forming load is approximately constant (in the range of 15 to $25 \mathrm{kN}$ ), there is a steep increase of the forming load (labelled as 'D') up to a peek value, corresponding to the sum of the individual critical instability loads of AISI304 and S460MC,

$$
P_{c r}=P_{c r}^{A I S I 304}+P_{c r}^{S 460 M C}=79+93.5=172.5 \mathrm{kN}
$$

The total plastic instability load $P_{c r}$ at which plastic instability is triggered is plotted in Fig. 9b as a dashed horizontal line labelled as ‘Total plastic instability’. Propagation of the plastic instability wave and locking by compression beading explains the subsequent valley and upward tail (labelled as 'E') of the load-displacement curve.

It is worth mentioning that only one valley is observed in Fig. 9b because the number of valleys in the load-displacement curve is related to the number of instability waves that are triggered. For example, the load-displacement curve of mode 3 (case 4 in Fig. 9a) 
contains two valleys before the final upward tail corresponding to locking by compression beading.

Experimental observations also show that there is no influence of the relative position between the counterfacing surfaces of AISI304 and S460MC tubes on the overall evolution of the load-displacement curve. This is a consequence of their critical instability loads being similar and allows concluding that it does not matter which tube is inside or outside when the slenderness ratio $l_{\text {gap }} / r_{0}$ is chosen from the intersection of the process windows that were previously determined for each of the individual AISI304 and S460MC tubes.

From what was mentioned above, the overall agreement between the experimental and the finite element predicted evolutions of the load-displacement curve is good taking into account the diversity and complexity of the phenomena that take place during endto-end joining of tubes of dissimilar materials by forming.

\subsection{A two stroke variant of the joining process}

The last section of the paper is focused on the challenge of joining tubes of dissimilar materials with very different strength by their ends. Fig. 10a shows two unsuccessful attempts performed with S460MC carbon steel $\left(\sigma_{Y}=460 \mathrm{MPa}\right)$ and AA6060-T6 aluminium $\left(\sigma_{Y}=160 \mathrm{MPa}\right)$ tubes.

As seen in the figure, the proposed process is not capable of joining the S460MC and AA6060-T6 tubes in a single stroke. This is because the expanded edge of the AA6060T6 tube is sheared along the circumferential direction while being forced against the chamfered end of the S460MC tube (left test case) or simply because both tubes experience a significant axial plastic deformation when the S460MC tube is forced against the chamfered end of the AA6060-T6 tube (right test case). 


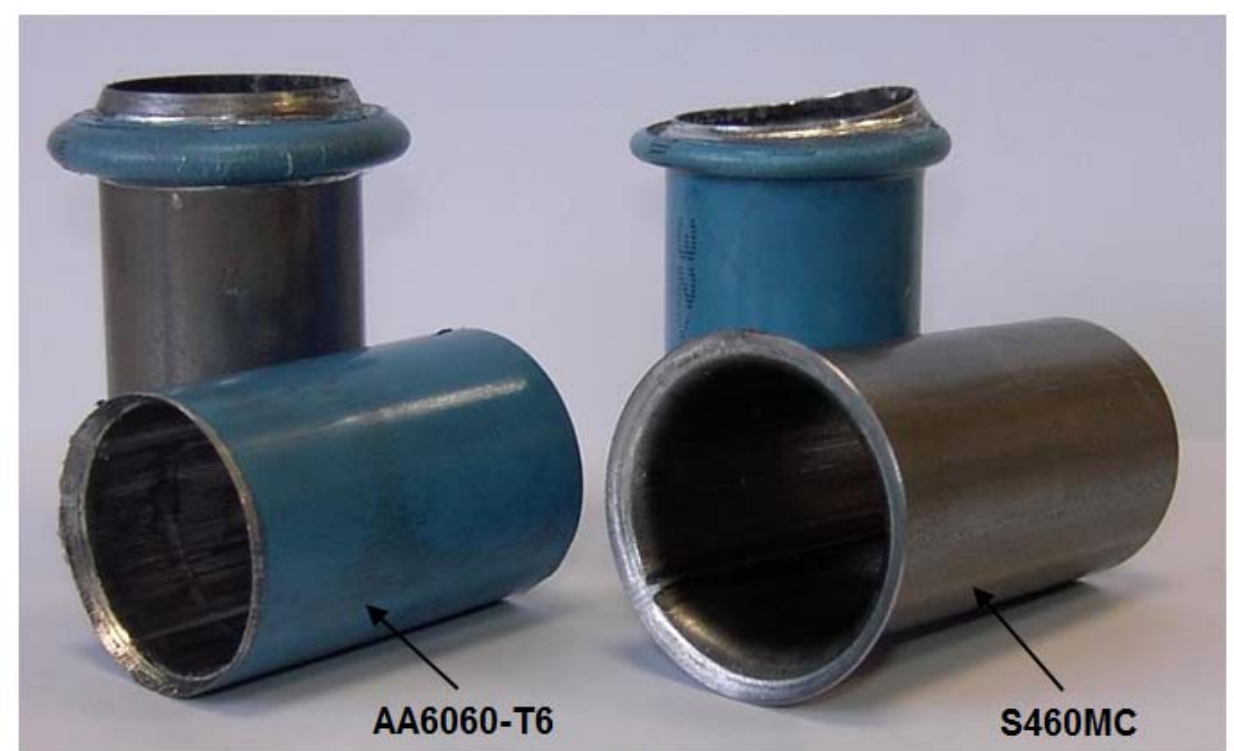

(a)
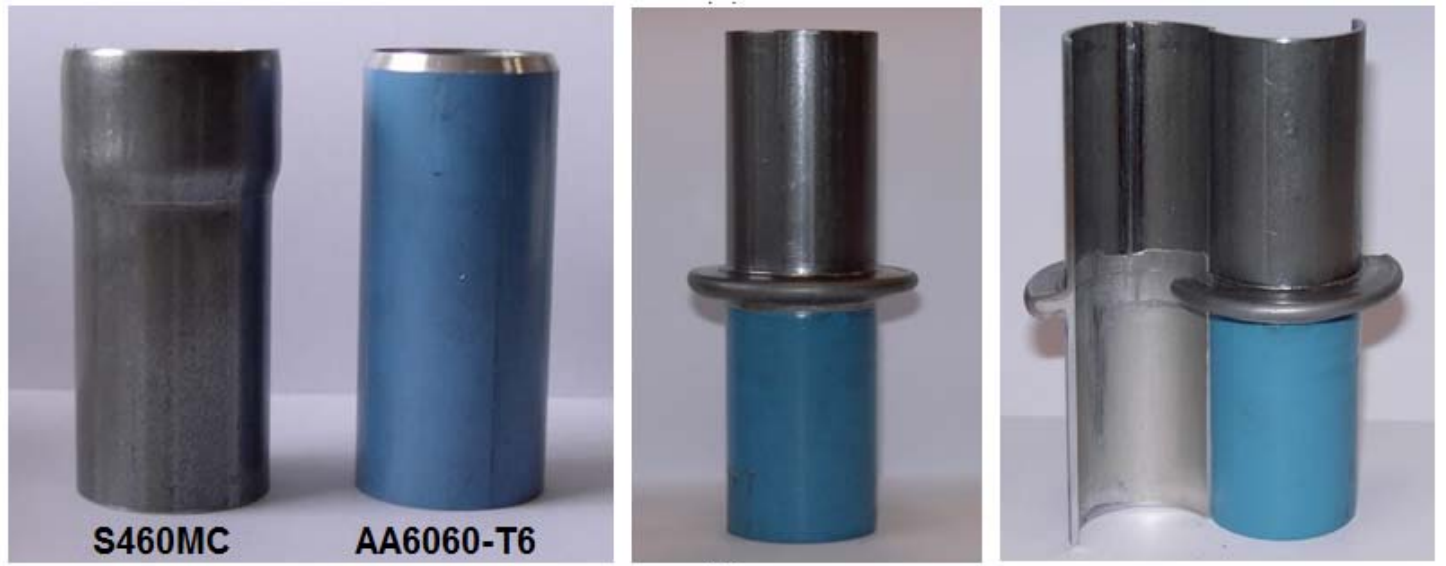

(b)

Fig. 10 - Joining end-to-end tubing of S460MC and AA6060-T6 by forming.

(a) Unsuccessful test cases performed with a single stroke approach;

(b) Successful test case performed with the two stroke variant of the joining process.

The alternative for successfully joining the S460MC and AA6060-T6 tubes by their ends is to replace the aforementioned single stroke procedure by a two stroke procedure in which the S460MC is previously expanded with a mandrel and subsequently assembled with the AA6060-T6 in order to ensure locking by compression beading (Fig. 10b). The two stroke solution is less flexible than the single stroke but it is capable of ensuring a sound joint between tubes made of dissimilar materials with very different strengths. 
To conclude it is worth noting that the two-stroke variant of the new proposed joining process requires preliminary expansion to be carried out in the tube with largest strength because otherwise its surface could be subsequently sheared by the chamfered edge of the inner tube. This problem can, however, be minimized if both tubes are used without chamfered edges because the two stroke variant of the new proposed joining process does not require the inner tube to behave as a tapered punch during the expansion stage. In fact, in contrast to single stroke joining, the expansion stage of the two stroke variant of the new proposed joining process is performed by means of a conventional mandrel.

\section{CONCLUSIONS}

Joining end-to-end tubing of dissimilar materials by forming can be performed by means of a simple, effective and environmental friendly solution that combines tube expansion and compression beading, at room temperature.

Combination of the process window of S460MC carbon steel and AISI304 stainless steel tubes allowed determining a slenderness ratio $l_{\text {gap }} / r_{0} \cong 1.9$ for successfully connecting both tubes in a single stroke, without experiencing inadmissible modes of deformation or failure by cracking along the plastically deformed weld seams. The process window was established under constant values of the reference radius $r_{0}$ of the tubes, of the thickness $t_{0}$ of the tube wall and of the angle $\alpha$ of the chamfered tube ends. Other geometries may require new characterization of the process windows by means of the methods and procedures that were described in this paper.

Experiments with S460MC carbon steel and AA6060-T6 aluminium tubes allowed concluding that joining end-to-end tubing of dissimilar materials with very different strengths require the utilization of a two stroke variant of the proposed joining process, in which the tube with largest strength should be preliminary expanded with a mandrel. 
As a result of this, the two stroke variant of the proposed joining process opens the possibility of connecting polymer and metal tubes at room temperature.

\section{ACKNOWLEDGMENTS}

The authors would like to acknowledge the support provided by Fundação para a Ciência e a Tecnologia of Portugal and IDMEC under LAETA - UID/EMS/50022/2013.

\section{REFERENCES}

[1] Mori KI, Bay N, Fratini L, Micari F, Tekkaya AE. Joining by plastic deformation. CIRP Annals of Manufacturing Technology 2013; 62: 673-694.

[2] Groche P, Wohletz S, Brenneis M, Pabst C, Resch F. Joining by forming - A review on joint mechanisms, applications and future trends. Journal of Materials Processing Technology 2014; 214: 1972-1994.

[3] Gonçalves A, Alves LM, Martins PAF. Tube joining by asymmetric plastic instability. Journal of Materials Processing Technology 2014; 214: 132-140.

[4] Gonçalves A, Alves LM, Martins PAF. Inclined tube-sheet plastically deformed joints. Steel Research International 2014; 85: 67-75.

[5] Alves LM, Dias EJ, Martins PAF. Processo de ligação de duas peças do travão de mão de um automóvel. Patent no. PT105554-A, 2012.

[6] Olson DL, Siewert TA, Liu S, Edwards GR (Editors). Welding, Brazing and Soldering. Metals Park, USA: ASM International; 1993.

[7] Adams RD, Adhesive Bonding - Science, Technology and Applications. Cambridge, UK: Woodhead Publishing; 2005.

[8] Budynas RG, Nisbett JK. Shigley’s Mechanical Engineering Design. New York, USA: McGraw Hill; 2008. 
[9] Zhang Q, Jin K, Mu D. Tube/tube joining technology by using rotary swaging forming method. Journal of Materials Processing Technology 2014; 214: 20852094.

[10] Psyk V, Rischa D, Kinsey BL, Tekkaya AE, Kleiner M. Electromagnetic forming A review. Journal of Materials Processing Technology 2011; 211: 787-829.

[11] Alves LM, Silva CMA, Martins PAF. End-to-end joining of tubes by plastic instability. Journal of Materials Processing Technology 2014; 214: 1954-1961.

[12] Alves LM, Medeiros JG, Martins PAF. Cold end forming of welded steel tubes. International Journal of Manufacturing, Materials and Mechanical Engineering 2011; 1: 18-30.

[13] Nielsen CV, Zhang W, Alves LM, Bay N, Martins PAF. Modeling of ThermoElectro-Mechanical Manufacturing Processes with Applications in Metal Forming and Resistance Welding. London, UK: Springer-Verlag; 2013.

[14] Cockroft MG, Latham DJ. Ductility and the workability of metals. Journal of the Institute of Metals 1968; 96: 33-39. 